\title{
Distribution State Estimation for Smart Distribution System
}

\author{
M. Karimi, Student Member, IEEE \\ H. Mokhlis, Member, IEEE \\ Department of Electrical Engineering \\ Faculty of Engineering, University of Malaya \\ Kuala Lumpur, 50603 Malaysia \\ mazaherkarimi@yahoo.com
}

\author{
A. H. A. Bakar, Member, IEEE \\ A. Shahriari, Student Member, IEEE \\ UM Power Energy Dedicated Advanced Centre (UMPEDAC) \\ Level 4, Wisma R\&D, University of Malaya \\ Kuala Lumpur, 59990 Malaysia \\ hazli@um.edu.my
}

\begin{abstract}
With the penetration of distributed generations into distribution network, more distribution automation will be required. Therefore, smart grid technologies become more important aspect in electric power system. Real-time monitoring of the network is a main step to achieve smart grid technologies. Hence, distribution state estimation becomes more essential for real-time monitoring of traditional distribution network. This paper addresses a distribution state estimation method to achieve smart grid technologies in a traditional distribution network. It will involve exploring distribution state estimation method with minimum number of real-time measurement devices. Therefore, pseudo measurements are applied to address the limitation of the number of real-time measurements. In addition, by incorporating load model, state estimation results are improved and enhanced. In this paper, dynamic aspect of distribution state estimation has been developed for smart grid purposes. The proposed distribution state estimation results are justified with PSCAD/EMTDC software to verify and validate the system state variables.
\end{abstract}

Index Terms-Distributed Generation, Distribution Automation, Distribution State Estimation, Smart Grid.

\section{INTRODUCTION}

Nowadays, the advances in monitoring equipment, communications and computing technologies have opened the opportunity for smart network development of distribution system. In addition to this, the continuous penetration of Distributed Generations (DGs) [1] also leads to the development of smart grid application for effectively control and operate the system. In the near future, the distribution system will be operating as an active network with penetration of DGs [1]. An active distribution network needs to use information and communication technologies to achieve smart grid concept. According to the Energy Department of United State, a smart grid uses information and communication technology to collect data and make a decision for best operation condition.

From smart grid point of view, system state monitoring is crucial and important. In the current practice, system state is obtained from Supervisory Control and Data Acquisition

This work was supported by postgraduate research fund (PPP) unit of University of Malaya and HIR/MOHE research grant, University of Malaya, Kuala Lumpur, Malaysia.
(SCADA) system. The control center of the network collects the system state of an electric power system through remote terminal units (RTUs), for the processing and decision making. Data transmitted to the control center should be accurate and reliable enough for controlling a power system. In order to have a reliable and accurate system state, the utilities need to upgrade their SCADA system and install more real-time measurements. However, the application of SCADA for distribution system is limited and only available for selected area. Moreover, in conventional operation of distribution network, real-time information is very limited to monitor the whole system at each point due to financial constraints. Hence, this issue could be addressed by using State Estimation (SE) method.

Ever since the introduction of State Estimation (SE) by F.C. Schweppe in the late of 1970 [2-4], various techniques have been proposed to solve a SE [5-9]. A statistical SE algorithm estimates the voltage magnitudes and angles and power flow at each node by using the real-time measurements, network data and load data [10]. A fundamental static state estimation algorithm by utilizing Weighted Least Square (WLS) method is presented in [11]. In this paper, WLS method is implemented based on singular value decomposition in contrast with the normal equation. A state estimator based on voltage and current waveform measurement, multiphase model and synchronized measurement is presented in [12]. Afterwards, the three phase state estimation is achieved by implementing least square method for radial distribution system [13].

Although there are various SE methods, they were not developed to address the limitation number of real-time measurement devices in traditional distribution network. Furthermore, most of the SE methods were developed for transmission system, which has plenty number of real-time measurement as compared to distribution system. Number and placement of real-time measurements are very important to identify the accuracy of estimation [14]. Implementation of SE in distribution network can be utilized for automation and control. Hence, enables an electric utility dispatcher to 
remotely monitor distribution components in a reliable and optimal mode [8].

Considering the limitation of the SE, this paper proposes an improved DSE method which will estimate an operation point of a traditional distribution system based on limited number of measurements. Pseudo measurements as bus power injections are applied in proposed DSE to solve the limitation number of measurements. Pseudo measurements data can be obtained from load forecasting, historical data or other approximation methods. In addition, customer loads such as domestics, commercials and industrials are modeled in this proposed method to calculate an accurate value of active and reactive power consumptions. The proposed scheme is implemented to a Malaysia's existing distribution network that consists of two small units of hydro generation connected in parallel to the grid. The proposed DSE has been validating in PSCAD/EMTDC software to test its ability for dynamic

$\begin{array}{lllll}\ddot{y} & \ddot{y} & 0 & \text { DISTRIBUTION } & \text { STATE Estimation }\end{array}$

\section{DistRIBUTION STATE ESTIMATION CONSTRAINS}

The continuous penetration of DGs in distribution system leads to the needs effective control and operate the system. To achieve this status, distribution network needs to use information and communication technologies to collect realtime data in order to make a decision for best operation condition. Therefore, utilities are expected to implement efficient monitoring devices to obtain an accurate state of the system. In order to develop distribution networks, many other automated meters, distribution SCADA and Distribution Management System (DMS) need to install in distribution level. Although, these technologies will be expected to setup in distribution level, the numbers of real-time measurement devices are very few due to high cost of these devices. On the other hand, the state estimation function of the transmission level cannot be directly implemented to the distribution level due to differing operation and structure philosophy. The major state estimation function differences between the transmission and distribution levels are summarized as follows:

$>$ The transmission network is an interconnected network with mesh topology, in contrast to the radial topology of a distribution network.

$>$ The number of monitoring devices in a distribution network for preparing real-time data is limited in contrast to a transmission network, so there are not sufficient devices to provide system observability.

$>$ The radial structure of a distribution network and low reactance/resistance ratio of the line create further limitations for the state estimation function.

According to these differences, the SE of the transmission level cannot be implemented into the distribution level. Therefore, the proposed DSE is introduced in this paper to estimate the system state of a distribution network.

\section{Proposed Distribution State Estimation Method}

The overall concept of proposed method is illustrated in Figure 1. It is presented the state estimation function at distribution network with their corresponding data acquisition.
The data acquisitions for distribution level are included realtime and pseudo measurements data.

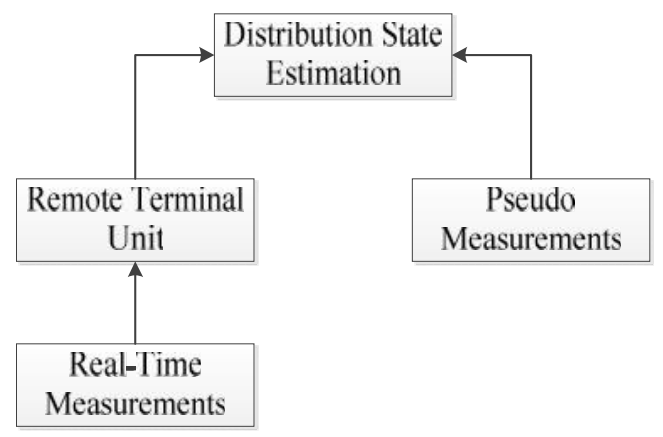

Figure 1. Distribution state estimation concept

The proposed SE flowchart for distribution network is illustrated in Figure 2. In the proposed method active and reactive power consumption will update within iterations. In this method, nodal voltage based for the DSE algorithm incorporating load modeling is proposed. It is different from the previous research in the following two major areas:

$>$ The sparsity problem of Jacobian matrix in traditional distribution network is solved by implementing customer load estimation or pseudo measurements as an active and reactive power injection at the system buses.

$>$ The proposed method incorporates load modeling to find the correct amount of active and reactive power which is related to voltage of the system. In reality, load values are dependent on voltage and system frequency.

In a power system including distribution and transmission system, the global measurement vector is indicated by $\boldsymbol{Z}$. For an $N$-bus power system with $m$ measurements, assume $r$ an error vector to be standard Gaussian that has a zero mean and variance $\sigma$. Let $i$ be the index of the measurements. The measurement equation is formulated in (1).

$$
Z_{i}=h_{i}(x)+r_{i}, \quad i=1,2, \ldots, m
$$

Where;

$\boldsymbol{Z}_{i}$ is the vector of measurements, $\boldsymbol{x}$ is the state vector, $\boldsymbol{h}_{i}(\boldsymbol{x})$ is the measurement matrix of the nonlinear function, $\boldsymbol{r}_{i}$ is the measurement errors vector.

Then, the measurement residual vector can be defined and formulated in (2).

$$
r_{i}=Z_{i}-h_{i}(x), \quad i=1,2, \ldots, m
$$

By applying the WLS method, it will minimize the objective function as describe in (3).

$$
J(x)=\sum_{i=1}^{m} \frac{\left(Z_{i}-h_{i}(x)\right)^{2}}{R_{i}}
$$

Where;

$R_{i}$ is diagonal matrix of $\left[1 / \sigma_{1}{ }^{2}, 1 / \sigma_{2}{ }^{2} \ldots 1 / \sigma_{\mathrm{m}}{ }^{2}\right], \sigma_{\mathrm{m}}{ }^{2}$ is the variance of the $m^{\text {th }}$ measurement error. 
The best solution of the state vector $\boldsymbol{x}$ can be achieved by the solution of the following equation;

$$
\begin{gathered}
{[G(x)]\left[\Delta x^{k+1}\right]=\left[H\left(x^{k}\right)\right][R]^{-1}\left[Z-h\left(x^{k}\right)\right]} \\
{[G(x)]=\left[H\left(x^{k}\right)\right]^{T}[R]^{-1}\left[H\left(x^{k}\right)\right]}
\end{gathered}
$$

It is calculated to reflect the expected accuracy of the corresponding meter. In (4), the $\boldsymbol{G}(\boldsymbol{x})$ is called the Gain matrix; it can be defined and formulated in (5). Solving the equation (4) is minimizing such errors and finds an optimal estimate; system state will be solved, iteratively. In this proposed method, WLS state estimation, as a mathematical analysis tools and an iterative solution, is presented to filter out such errors and find an optimal estimate of system states in distribution network.

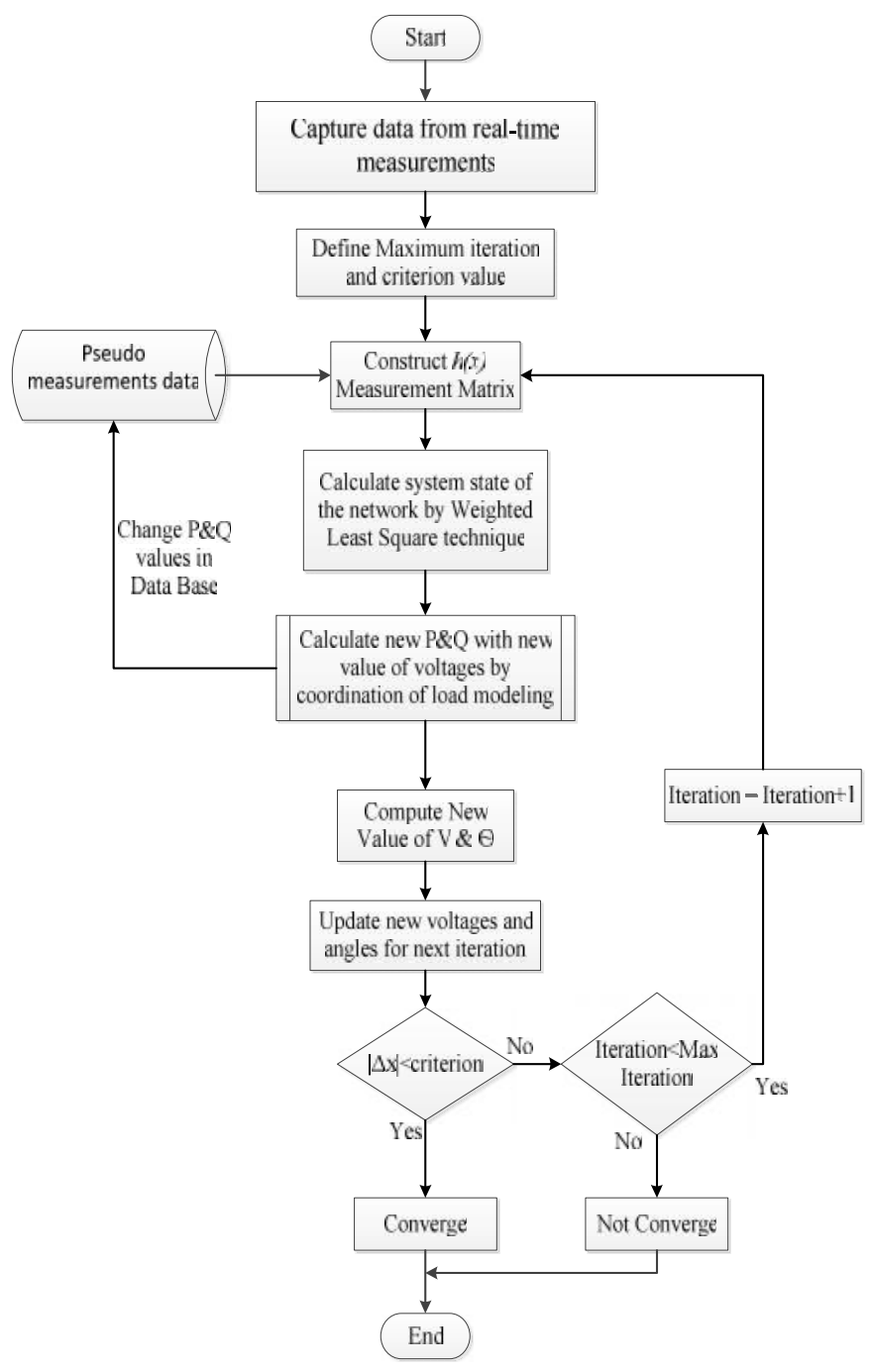

Figure 2. Proposed state estimation for distribution network
The proposed DSE method is based on voltage measurements. The calculated values are used to construct the measurement matrix, $\boldsymbol{h}(\boldsymbol{x})$, in order to solve the (4). Since realtime information is very limited in a conventional distribution system, the state estimator cannot attain convergence. Therefore, pseudo measurements are needed to assist convergence of the state estimator for distribution network. The pseudo measurements are used at some buses where measurements not available.

In the proposed algorithm, the entire static load model can be implemented as presented in (6) and (7).

$$
\begin{gathered}
P_{i}=P_{i_{0}}\left(\frac{\left|V_{i}\right|}{\left|V_{i_{0}}\right|}\right)^{a}, \quad i=1,2, \ldots, m \\
Q_{i}=Q_{i_{0}}\left(\frac{\left|V_{i}\right|}{\left|V_{i_{0}}\right|}\right)^{b}, \quad i=1,2, \ldots, m
\end{gathered}
$$

In these equations, $P_{i 0}$ and $Q_{i 0}$ is the nominal power at the rated voltage. For this model, $P_{i 0}$ and $Q_{i 0}$ are normally taken as the values at initial system operating condition.

In the proposed DSE, the load values are updated during every iteration. When the proposed DSE converge, the obtained state of the system can be used for smart grid applications such as islanding operation and energy management.

\section{TEST SYSTEM}

The test system is illustrated in Figure 3. It consists of 34 buses, 26 lumped loads with 2 mini-hydro generators of 2 MVA with 2.5 (s) of inertia constant are operated at $3.3 \mathrm{kV}$. Both mini-hydro generators are connected on main bus bar and supplies power to distribution network. The mini-hydro generators are connected to the distribution network through two parallel units of 2 MVA transformers to step up the voltage level to $11 \mathrm{kV}$. The distribution network is connected to grid through a step down transformer rated $132 \mathrm{kV} / 11 \mathrm{kV}$. This test system is modeled using PSCAD/EMTDC.

The DSE is based on three measurements at main substation to measure active and reactive power flow. In this test system, the outputs of the main feeders are measured for state estimation purposes. These feeders are connected to the bus numbers 1 to 31,30 to 13 and 30 to 27 . The real-time measurements are installed to monitor the voltages at bus 30 and bus 31 . The real-time measurements also monitored the active and reactive power injections of the aforementioned feeders. The measurement accuracy is assumed to be $1 \%$, which is based on practical data [14]. For other buses, pseudo measurement (based on historical data) data with $25 \%$ error are used to estimate power injection. 


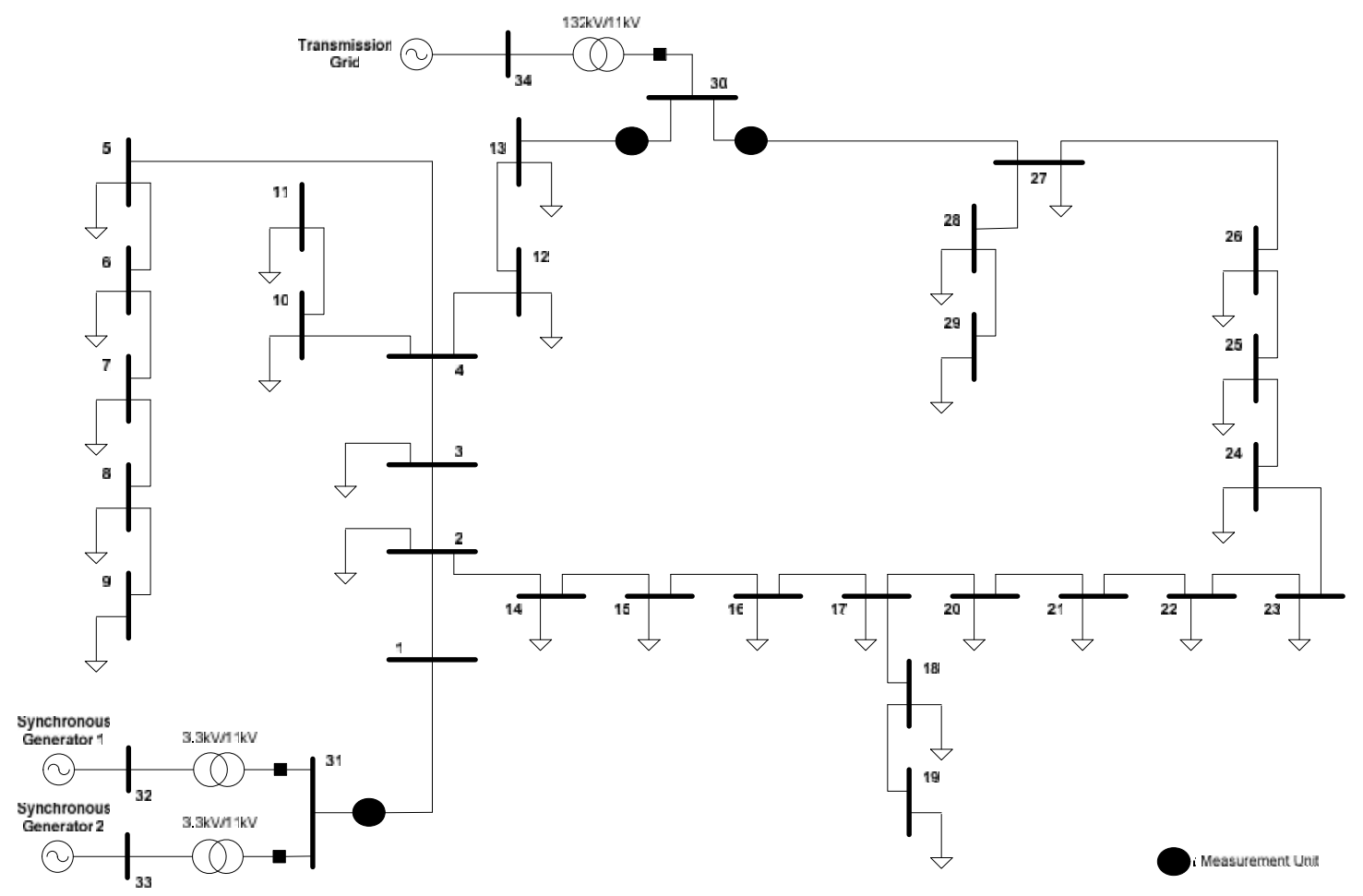

Figure 3. Distribution network connected with mini-hydro

\section{SiMULATION RESUlTS}

In this section, the results of proposed DSE method and real-time measurements data are compared. The simulation study was carried out in two different conditions; 1) Steady state analysis and 2) Dynamic by varying some loads. In this simulation, static load model is applied to model all load in the system. It should be noted that the actual value is shown by dashed line style and the estimated value is presented by solid line style.

\section{1) Steady State Condition}

In this case study, the distribution network is in a steady state condition. Table I shows some example of the actual and estimated value obtained from the proposed DSE. In general it can be observed that the value is of the actual and estimated is close to each other. The overall estimated voltage magnitude and angle are presented in Figure 4 and Figure 5, respectively. The result obtained from PSCAD/EMTDC software is also plotted in the same figures to compare the accuracy of the estimated state.

In general, it can be observed that the estimated value of DSE is close to the actual value with small variation. For voltage magnitude and angle accuracy, the average variation error of overall buses are 0.003959 and 0.005948 respectively. Exception is for bus 1, where the estimated state is equal with the PSCAD/EMTDC value. This occurs due to real-time measurement device is installed in this bus. Furthermore, bus number 1 is selected as reference bus. Different scenario however occurs at bus 30 although real-time measurement is installed. There is a small difference between estimated with actual value, which are $0.4042 \%$. The reason behind this is that the algorithm tries to minimize the overall error at each bus as describe in (3). Nevertheless, for practical application the measurement value should be used instead of the estimated one.

TABLE I. Actual AND Estimated VALUE OF SOME Buses

\begin{tabular}{ccccc}
\hline \hline \multirow{2}{*}{$\begin{array}{c}\text { Bus } \\
\text { Number }\end{array}$} & \multicolumn{2}{c}{ Voltage (P.U) } & \multicolumn{2}{c}{ Angles (Radians) } \\
\cline { 2 - 5 } $\mathbf{1}$ & Actual & Estimated & Actual & Estimated \\
\hline \hline $\mathbf{2}$ & 1.0264 & 1.0264 & 0.0000 & 0.0000 \\
\hline $\mathbf{4}$ & 0.9974 & 1.0001 & -0.0698 & -0.0748 \\
\hline $\mathbf{5}$ & 0.9861 & 0.9900 & -0.0546 & -0.0636 \\
\hline $\mathbf{6}$ & 0.9842 & 0.9885 & -0.0438 & -0.0434 \\
\hline $\mathbf{8}$ & 0.9824 & 0.9870 & -0.0337 & -0.0312 \\
\hline $\mathbf{1 0}$ & 0.9801 & 0.9850 & -0.0226 & -0.0131 \\
\hline $\mathbf{1 2}$ & 0.9860 & 0.9901 & -0.0547 & -0.0619 \\
\hline $\mathbf{1 3}$ & 0.9851 & 0.9891 & -0.0620 & -0.0745 \\
\hline $\mathbf{1 6}$ & 0.9812 & 0.9853 & -0.1126 & -0.1358 \\
\hline $\mathbf{1 8}$ & 0.9861 & 0.9895 & -0.0342 & -0.0351 \\
\hline $\mathbf{1 9}$ & 0.9789 & 0.9827 & -0.0069 & -0.0059 \\
\hline $\mathbf{2 1}$ & 0.9784 & 0.9823 & -0.0029 & 0.0023 \\
\hline $\mathbf{2 2}$ & 0.9746 & 0.9785 & -0.0025 & -0.0044 \\
\hline $\mathbf{2 3}$ & 0.9723 & 0.9767 & -0.0103 & -0.0150 \\
\hline $\mathbf{2 4}$ & 0.9714 & 0.9760 & -0.0152 & -0.0183 \\
\hline $\mathbf{2 5}$ & 0.9712 & 0.9758 & -0.0237 & -0.0303 \\
\hline $\mathbf{2 6}$ & 0.9711 & 0.9757 & -0.0311 & -0.0378 \\
\hline $\mathbf{2 8}$ & 0.9711 & 0.9758 & -0.0378 & -0.0430 \\
\hline $\mathbf{2 9}$ & 0.9707 & 0.9755 & -0.0406 & -0.0448 \\
\hline $\mathbf{3 0}$ & 0.9704 & 0.9752 & -0.0379 & -0.0435 \\
\hline \hline & & 0.9852 & -0.1136 & -0.1406 \\
\hline \hline
\end{tabular}




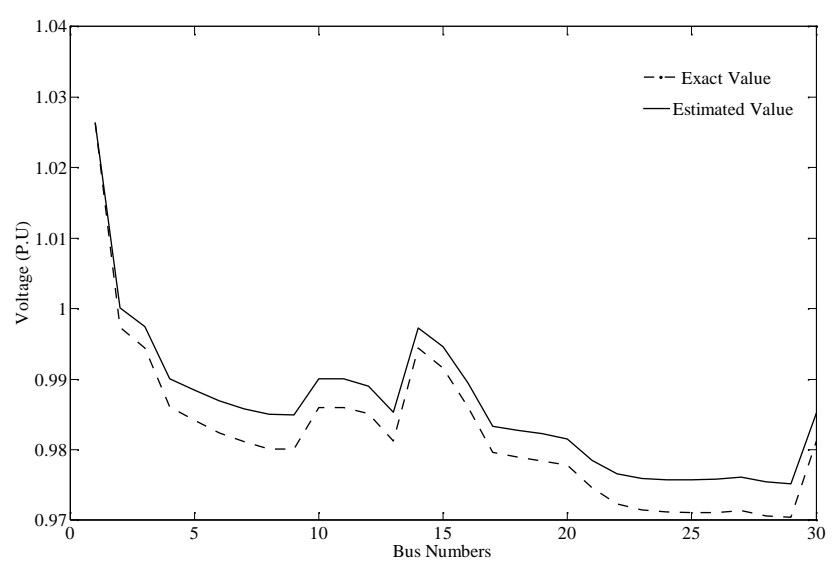

Figure 4. Actual and estimated value of voltage magnitude

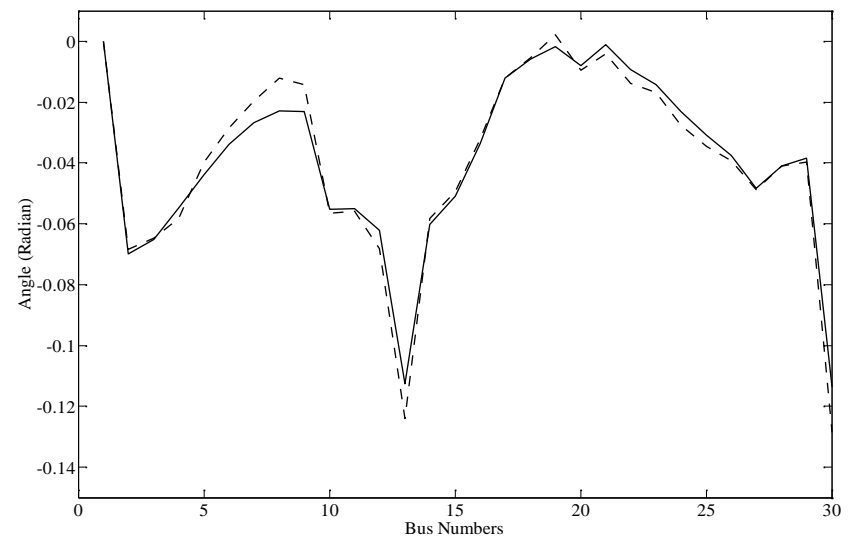

Figure 5. Actual and estimated value of voltage angles

Figure 6 and Figure 7 shows the comparison between actual and estimated values for active and reactive power load at each bus, respectively. Overall the estimated value of proposed method is closely followed the actual value. The average variation error for active and reactive powers are 0.015056 and 0.011209 , respectively.

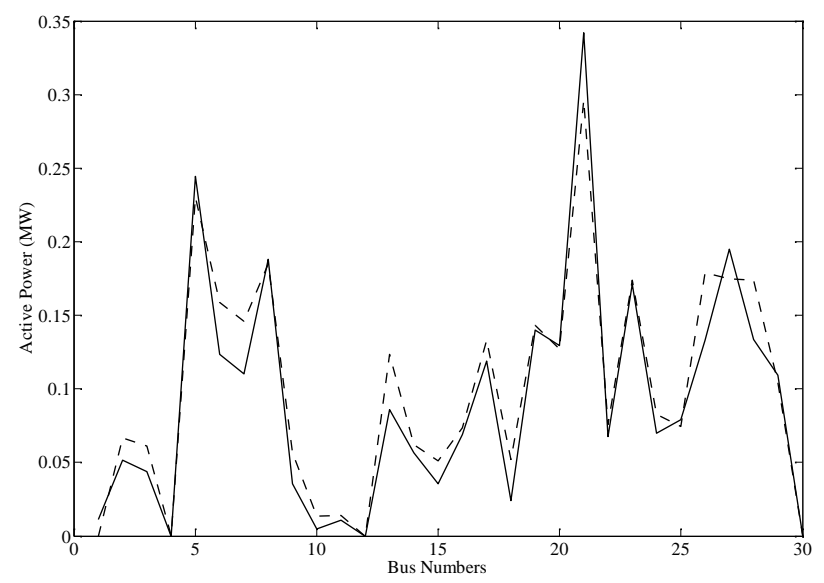

Figure 6. Actual and estimated value of active power

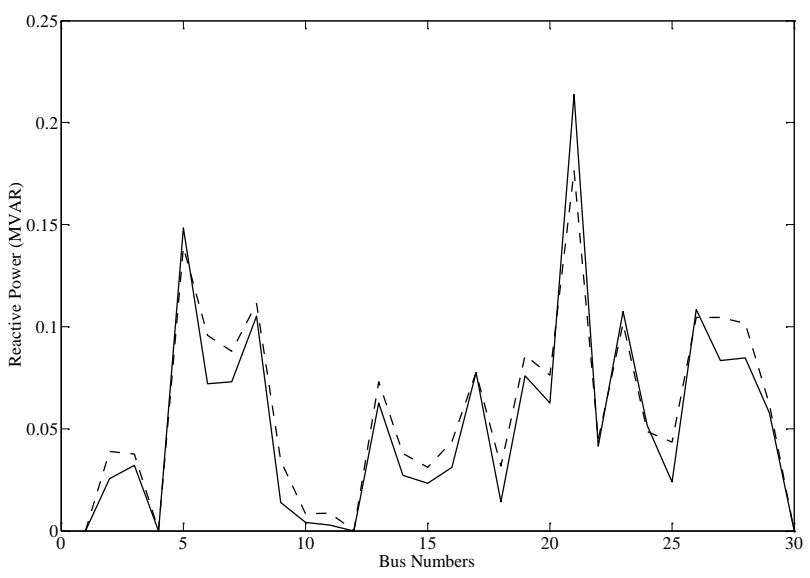

Figure 7. Actual and estimated value of reactive power

\section{2) Dynamic Condition}

For the test system considered, sudden changes in system loads is simulated. Load values are illustrated in Table II. Active power estimated of DSE method and active power data of real-time measurements are presented in this table. These data have been presented for load changes in distribution network. Bus numbers 5, 6, 7, 8, 9 and 19 have been selected randomly in order to show the effectiveness of proposed DSE method for dynamic conditions. Estimated and actual values of active power consumption are illustrated before load changing and after load changing. Active power loads at aforementioned buses are changed at time $t=3$ seconds.

The value of active power errors in percentage for each bus is also calculated in Table II. It can be observed that the variation of error value is small before and after load changing.

TABLE II. PERformance ANALysis of DSE Method

\begin{tabular}{|c|c|c|c|c|c|}
\hline \multirow[b]{2}{*}{ Bus } & \multirow[b]{2}{*}{$\begin{array}{l}\text { Measuring } \\
\text { Methods }\end{array}$} & \multicolumn{2}{|c|}{$t<3(\mathrm{~s})$} & \multicolumn{2}{|c|}{$t>\mathbf{3}(\mathrm{s})$} \\
\hline & & $\begin{array}{c}\text { Load } \\
\text { Values } \\
(\mathrm{MW})\end{array}$ & $\begin{array}{c}\text { Error in } \\
\text { Percentage }\end{array}$ & $\begin{array}{c}\text { Load } \\
\text { Values } \\
(\mathrm{MW})\end{array}$ & $\begin{array}{c}\text { Error in } \\
\text { Percentage }\end{array}$ \\
\hline \multirow[b]{2}{*}{5} & 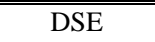 & 0.2505 & \multirow[b]{2}{*}{-2.03} & 0.2435 & \multirow[b]{2}{*}{-1.83} \\
\hline & Real-time & 0.2302 & & 0.2252 & \\
\hline \multirow[b]{2}{*}{6} & DSE & 0.1283 & \multirow[b]{2}{*}{3.04} & 0.1213 & \multirow[b]{2}{*}{3.42} \\
\hline & Real-time & 0.1587 & & 0.1555 & \\
\hline \multirow[b]{2}{*}{7} & DSE & 0.1146 & \multirow[b]{2}{*}{3.14} & 0.1076 & \multirow[b]{2}{*}{3.55} \\
\hline & Real-time & 0.146 & & 0.1431 & \\
\hline \multirow[b]{2}{*}{8} & DSE & 0.1928 & \multirow[b]{2}{*}{-0.8} & 0.1858 & \multirow[b]{2}{*}{-0.47} \\
\hline & Real-time & 0.1848 & & 0.1811 & \\
\hline \multirow[b]{2}{*}{9} & DSE & 0.0389 & \multirow[b]{2}{*}{1.75} & 0.0319 & \multirow[b]{2}{*}{2.33} \\
\hline & Real-time & 0.0564 & & 0.0552 & \\
\hline \multirow[b]{2}{*}{19} & DSE & 0.1275 & \multirow[b]{2}{*}{1.55} & 0.1196 & \multirow[b]{2}{*}{2.06} \\
\hline & Real-time & 0.143 & & 0.1402 & \\
\hline
\end{tabular}


Figure 8 shows an active power changing at selected buses. In this figure, the behavior of active power of DSE method and real-time measurement for duration of simulation study is presented.

It should be noted that the execution time periods of the proposed DSE are equal to 0.009467 seconds using a personal PC (3.06 GHz CPU, 3 GB RAM).

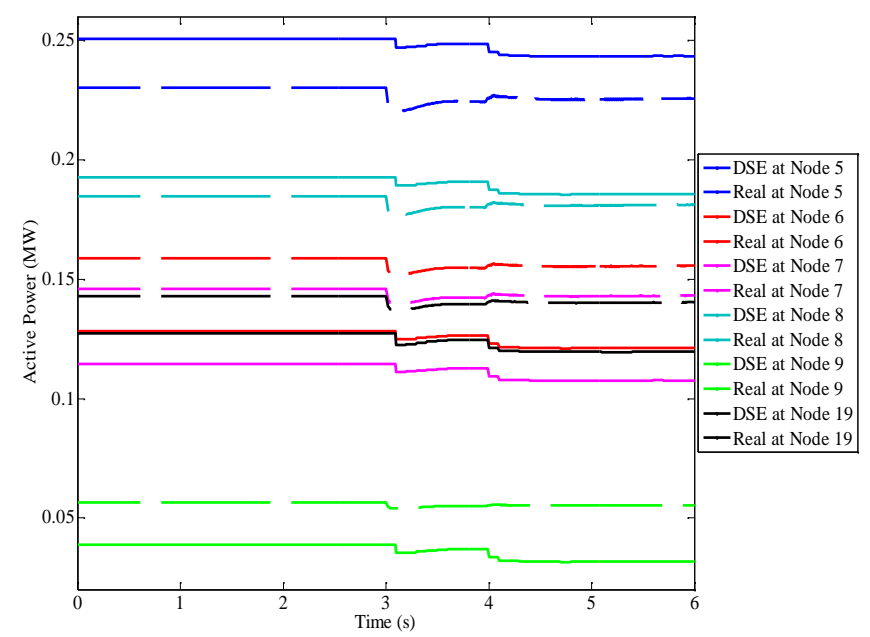

Figure 8. Comparison between estimated and actual value of active power

\section{CONCLUSION AND DISCUSSION}

This paper has presented a distribution state estimation method based on combination of real-time and pseudo measurement devices for conventional distribution system. It applied state estimation to obtained system state variables with very few numbers of measurements. The proposed method is useful to monitor conventional distribution network, which has limited real time measurements. The effectiveness of proposed method has been validated for steady state and dynamic conditions. From the simulation results, it can be observed that the estimated value of proposed method is very close to actual value. This application of state estimation technique made the proposed scheme economical for practical implementation in any distribution networks. Furthermore, a smart grid application for distribution system with limited number of real time measurements could be also implemented.

\section{ACKNOWLEDGMENT}

This work was supported by University of Malaya, Kuala Lumpur under postgraduate research fund (PPP) Grant (Grant code: PS009-2012A) and HIR/MOHE research Grant (Grant code: D000004-16001).

\section{REFERENCES}

[1] A. P. S. Meliopoulos, et al., "Smart grid technologies for autonomous operation and control," Smart Grid, IEEE Transactions on, vol. 2, pp. $1-10,2011$.

[2] F. C. Schweppe and J. Wildes, "Power System Static-State Estimation, Part I: Exact Model," Power Apparatus and Systems, IEEE Transactions on, vol. PAS-89, pp. 120-125, 1970.

[3] F. C. Schweppe and D. B. Rom, "Power system static-state estimation, Part II: Approximate model," Power Apparatus and Systems, IEEE Transactions on, pp. 125-130, 1970.

[4] F. C. Schweppe, "Power System Static-State Estimation, Part III: Implementation," Power Apparatus and Systems, IEEE Transactions on, vol. PAS-89, pp. 130-135, 1970.

[5] F. F. Wu, "Power system state estimation: a survey," International Journal of Electrical Power \& Energy Systems, vol. 12, pp. 80-87, 1990.

[6] M. Filho, et al., "Bibliography on power system state estimation(19681989)," IEEE Transactions on Power Systems, vol. 5, pp. 950-961, 1990.

[7] A. Bose and K. A. Clements, "Real-time modeling of power networks," Proceedings of the IEEE, vol. 75, pp. 1607-1622, 1987.

[8] A. Gomez-Exposito, et al., "A multilevel state estimation paradigm for smart grids," Proceedings of the IEEE, vol. 99, pp. 952-976, 2011.

[9] M. KARIMI, et al., "Distribution State Estimation Incorporating Load Modelling," PRZEGLAD ELEKTROTECHNICZNY, vol. 88, pp. 166169, 2012.

[10] H. Yih-Fang, et al., "State Estimation in Electric Power Grids: Meeting New Challenges Presented by the Requirements of the Future Grid," Signal Processing Magazine, IEEE, vol. 29, pp. 33-43, 2012.

[11] C. Madtharad, et al., "Power system state estimation using singular value decomposition," Electric power systems research, vol. 67, pp. 99-107, 2003.

[12] A. P. S. Meliopoulos, et al., "Power system state estimation: modeling error effects and impact on system operation," in System Sciences, 2001. Proceedings of the 34th Annual Hawaii International Conference on, 2001, pp. 682-690, 2001.

[13] M. E. Baran and A. W. Kelley, "State estimation for real-time monitoring of distribution systems," Power Systems, IEEE Transactions on, vol. 9, pp. 1601-1609, 1994.

[14] R. Singh, et al., "Measurement placement in distribution system state estimation," Power Systems, IEEE Transactions on, vol. 24, pp. 668675, 2009. 